\title{
The Fermentation of Trehalose by Yeasts and its Taxonomic Implications
}

\author{
By R. J. BOUTHILET, NORA E. NEILSON*, E. M. MRAK \\ AND H. J. PHAFF \\ Division of Food Technology, University of California, Berkeley
}

\begin{abstract}
SUMMARY: In the classification of yeasts it is customary to use an infusion of bakers' yeast as the basal medium for fermentation tests. This extract frequently contains variable amounts of trehalose. A number of yeasts were observed to ferment yeast extract and trehalose. The fermentation of yeast extract is serious from a taxonomic point of view, since it gives the impression of positive fermentation of a sugar which actually may not be fermentable. Dilute yeast autolysate should be used as the basal fermentation medium since during autolysis trehalose is destroyed. The fermentation of yeast extract (without added sugar) is easily observed in Durham tubes by the collection of gas in the inserts, but when Einhorn fermentation tubes are used gas production is seldom apparent.
\end{abstract}

One culture (N-18) isolated from spoiled apricots, and identified as Candida tropicalis showed adaptive trehalose fermentation.

The ability of various yeasts to ferment trehalose was investigated, using 133 cultures, representing twenty genera and seventy-three species. Sixteen species representing seven genera fermented yeast extract and trehalose.

The fermentation of trehalose is worthy of consideration as a character for use in differentiating certain species of Candida, and perhaps other yeasts.

In identifying yeasts it is necessary to use a number of natural media the compositions of which are uncertain. Although the method of preparation may be well standardized, these natural media undoubtedly vary in composition from batch to batch. These variations were considered of sufficient importance by Bedford (1942) to warrant the use of a chemically defined medium rather than liquid wort for studying cell and film characteristics of Hansenula spp. The use of chemically defined media has not been extended to the other genera of yeasts, principally because most yeasts grow poorly in such media, unless fortified with growth factors. Stelling-Dekker (1931) used fermentation media prepared by adding $2 \%$ of a given sugar to a water extract of bakers' yeast. This method has also been used for a number of years in our laboratory.

During recent investigations it was observed that a strain $\mathrm{N}-18$, isolated from home-canned apricots, and later identified as Candida tropicalis, appeared to ferment both maltose and lactose when the yeast-extract medium of StellingDekker (1931) was employed in Durham fermentation tubes. In view of the observation of Kluyver (1931), that in general, yeasts fermenting maltose do not ferment lactose and vice versa, this finding seemed of interest. Custers's (1940) report, that Brettanomyces claussenii fermented maltose and lactose, is the only known exception to this rule. B. claussenii also ferments maltose and lactose when the auxanographic plate method of Lodder (1934) is used; but culture N-18 was found to ferment maltose and not lactose.

* Department of Dairying, University of British Columbia, Vancouver, B.C. 
In the past three years, occasional strains of yeast in this laboratory have fermented maltose strongly and apparently fermented lactose weakly. The majority of these strains were classed as belonging to the genus Candida. When these strains and strain N-18 were grown in yeast-extract medium without added carbohydrate, fermentation to a similar degree as that of lactose occurred, indicating the presence of a fermentable substance in the basal medium. To our knowledge only one similar observation has been reported in the literature. Sacchetti (1933) observed fermentation of plain yeast extract by certain Torulopsis species, but he did not attempt to explain the phenomenon. It appears quite possible that in the past fermentation tests with other common sugars have incorrectly been termed positive in cases when gas might have been produced from the plain yeast extract instead of the added sugar.

Bakers' yeast is known to contain variable amounts of the disaccharide trehalose, sometimes as high as $14 \%$ of the dry-weight (Myrbäck \& Örtenblad, 1936). Trehalose was first found in American bakers' yeast by Koch \& Koch (1925), and later in Dutch bakers' yeast by Boesecken (see Kluyver \& van Roosmalen, 1932), in French bakers' yeast by Tanret (1931), again in America by Steiner \& Cori (1935) and in Sweden by Myrbäck (1937). Brandt (1941) has shown that although bakers' yeast grown under aerobic conditions stores considerable quantities of trehalose, it stores none under anaerobic or semi-anaerobic conditions. It seemed likely that the trehalose of bakers' yeast might be extracted during the preparation of yeast extract and would be responsible for the observed fermentation of plain yeast extract. This paper deals with the nature of the fermentable material in bakers' yeast extract (Stelling-Dekker), the types of yeast which are able to ferment it and its possible significance in yeast taxonomy.

\section{EXPERIMENTAL}

Methods. Two basal media were employed for fermentation studies, yeast extract and yeast autolysate. The yeast extract (Stelling-Dekker, 1931) was prepared by autoclaving a suspension of $200 \mathrm{~g}$. of bakers' yeast in $1 \mathrm{l}$. water for $15 \mathrm{~min}$. under 15 pounds pressure, and filtering, first while hot and again when cold. The extract may be easily clarified with fresh egg-albumin, or with a filter-aid. The yeast autolysate was made by incubating equal weights of bakers' yeast and water at $55^{\circ}$ for $72 \mathrm{hr}$., then bringing the mixture to a boil and filtering with the aid of 'filter-cel'. The yeast extract was used undiluted, and the autolysate diluted with nine parts of water. Except where noted, fermentations were carried out in small Durham fermentation tubes. When required, $2 \%$ of the desired carbohydrate was added to the basal medium.

\section{Isolation and identification of the fermentable substance in the yeast extract}

In order to determine the nature of the fermentable substance present in yeast extract prepared from bakers' yeast, a vacuum concentrate $(5: 1)$ of the extract was fractionated with increasing concentrations of ethanol and the precipitates formed were each made up to original volume with water. The last 
fraction, containing the material soluble in $70 \%(\mathrm{v} / \mathrm{v})$ ethanol, was evaporated in vacuo and the residue made up to volume, as were the precipitates. A sample of each of the fractions was used as a medium for fermentation tests with strain $\mathrm{N}-18$. Only the fraction soluble in $70 \%$ ethanol produced gas, indicating that the fermentable substance was probably a simple carbohydrate.

The unknown material was obtained in quantity from vacuum-dried yeast extract by the methods for the isolation and purification of sugars as outlined by Morrow \& Sandstrom (1935). The material was non-reducing, and was proved by specific rotation and other characteristics to be identical with trehalose. Strain N-18, and other strains forming gas in yeast extract, fermented the isolated trehalose, thus indicating that it was responsible for the gas production in yeast extract.

Effect of the type of fermentation apparatus. That previous workers using yeast extract as the basal fermentation medium (Stelling-Dekker, 1931; Lodder, 1934; Diddens \& Lodder, 1942) have not reported the presence of a fermentable substance or the apparent combined fermentation of maltose and lactose may be due to the type of fermentation tube employed. The Dutch workers use Einhorn tubes for routine work. To clarify this point of difference, comparative fermentation tests with a number of trehalose-fermenting strains were made on the same batch of yeast-extract medium in Einhorn tubes, large Durham tubes (Henrici, 1941) and small Durham tubes.

There was gas production in all Durham tubes but none in the Einhorn tubes. Mixing of the contents of the Einhorn tubes after several days' growth as recommended by the Dutch workers, in order to give greater sensitivity, resulted in only two positives out of sixteen strains which were positive in Durham tubes. These findings confirm the above hypothesis, indicate the lesser sensitivity of the Einhorn tubes, and suggest that when Durham tubes are used another basal fermentation medium should be employed.

Neither time nor method of heating yeast in water during the preparation of yeast extract appear to be important factors, since steaming at $100^{\circ}$ for $10 \mathrm{~min}$. or autoclaving at $120^{\circ}$ for $5,10,15,25$, or $40 \mathrm{~min}$., all gave similar results when fermentation tests were made with the resultant extracts. The concentration of the extract is important, since as a result of differences in manufacture and yeast strain used, the trehalose content of bakers' yeast varies greatly. In one instance, yeast extract (Stelling-Dekker) showed gas production in $72 \mathrm{hr}$. with strain N-18. When this yeast extract was diluted to half strength, 4 days were required for gas production, whereas growth but no gas production was noted when the dilution was greater than $\mathbf{1 / 4}$. Concentration of the original extract, on the other hand, shortened the time taken for gas formation to appear.

\section{Adaptive fermentation of trehalose}

When strain N-18 was grown on yeast-extract agar instead of wort agar before inoculation into yeast-extract fermentation tubes, gas production was greater and more rapid, suggesting an adaptive mechanism. Similar results were obtained when the organism was grown on a medium containing trehalose.

Experiments conducted with the Warburg respirometer in anaerobic con- 
ditions and the washed-cell technique confirmed our findings (see Fig. 1). Strain $\mathrm{N}-18$ was grown on a medium containing $10 \%$ of yeast autolysate and $1 \%$ added glucose or trehalose in Kolle flasks at $30^{\circ}$ for $24 \mathrm{hr}$. Strain N-18 grown in $1 \%$ glucose was unable to adapt itself to trehalose fermentation under

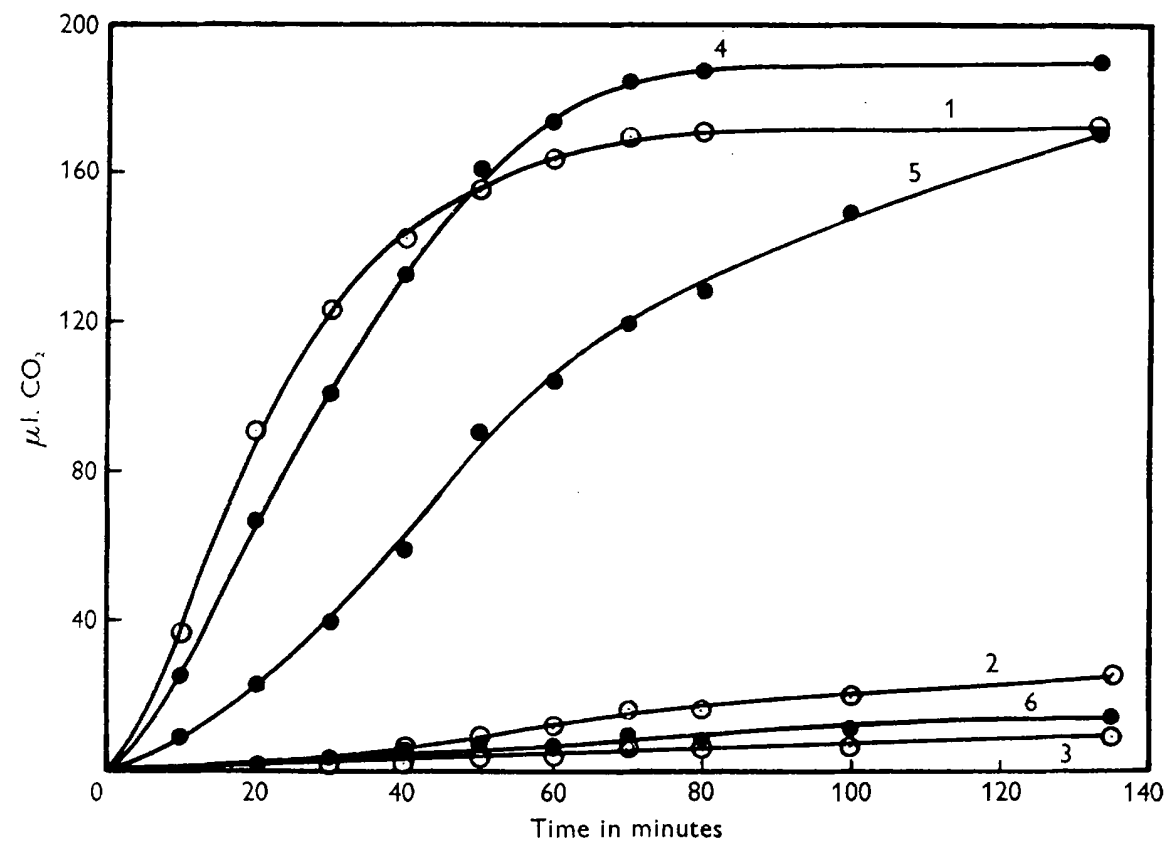

Fig. 1. 'The adaptive fermentation of trehalose by Candida tropicalis. (1) Glucose-grown cells, $5 \mu \mathrm{mol}$. of glucose; (2) glucose-grown cells, $2 \cdot 5 \mu \mathrm{mol}$. trehalose; (3) glucose-grown cells, endogenous; (4) trehalose-grown cells, $5 \mu \mathrm{mol}$. glucose; (5) trehalose-grown cells, $2 \cdot 5 \mu \mathrm{mol}$. trehalose; (6) trehalose-grown cells, endogenous. Temperature $30^{\circ} ; \mathrm{M} / 30$ $\mathrm{KH}_{2} \mathrm{PO}_{4}$ buffer. $\mathrm{O}-\mathrm{O}$, glucose-grown cells; $-\mathrm{O}-$, trehalose-grown cells.

anaerobic conditions within $2 \frac{1}{2} \mathrm{hr}$. after contact with the new substrate. On the other hand, when the culture was grown in the presence of trehalose, it fermented the trehalose at almost the same rate as it did glucose. Essentially, similar results were obtained using the van Iterson-Kluyver fermentometer (see Stelling-Dekker, 1931), in which conditions are also anaerobic and little or no cell multiplication occurs. With this technique the observations were extended over a period of about $\mathbf{2 4} \mathrm{hr}$.

This yeast N-18 is, as far as we know, the first reported to show a distinct adaptive trehalose fermentation. Previously it was indicated (Rhoades, 1941) that a beer yeast (of doubtful identity, since it apparently could not ferment maltose under any conditions) and a strain of Saccharomyces cerevisiae fermented trehalose when the cells were grown on galactose, maltose, melezitose or $\alpha$-methylglucoside, but not when grown on glucose. Rhoades used Lindner's well-slide technique to test fermentation. His paper, however, leaves the impression that it would be desirable to repeat some of the experiments before accepting the conclusions. 


\section{Recommended basal medium}

The problem of preparing a basal medium free of fermentable sugar was overcome by using a diluted yeast autolysate (as described above) rather than a water extract. Myrbäck \& Örtenblad (1937) demonstrated the enzyme trehalase in yeast autolysate and on the cell debris, and showed that the glucose formed by hydrolysis was fermented during autolysis. This explains why the stored trehalose disappears during autolysis of baker' yeast.

Another method of obtaining a basal yeast-extract medium free of trehalose is to use brewers' yeast instead of bakers' yeast. Müntz (1876) sought trehalose in brewers' yeast because of its presence in other fungi, but was unable to detect it. Myrbäck \& Örtenblad (1937) also found no trehalose in brewers' yeast, an observation we were able to confirm with two different commercial samples of American brewers' yeast. The extracts of these samples failed to show gas production with strain $\mathbf{N}-18$, unless a fermentable sugar was added.

\section{Fermentation of trehalose and yeast extract by various yeasts}

The study of the trehalose fermentation was extended in the hope that some of the results might have taxonomic application. The first trials indicated that only a few yeasts belonging to the genus Candida were strong trehalose fermenters, although none of the stock cultures tried compared in rate with strain N-18 (identified as C. tropicalis).

If the use of trehalose fermentation would help the classification of Candida species or other yeasts it would well justify the addition of another sugar to those already used in routine fermentation and utilization tests. Several workers have tested yeasts and other fungi for trehalose fermentation. Bau (1899) reported that trehalose is fermented by Saccharomyces ellipsoideus, $S$. pastorianus and Monilia candida, but very little by the 'Apiculate yeasts' or Schizosaccharomyces. Lindner (1887) found other yeasts capable of fermenting trehalose, namely Monilia candida, Saccharomyces anomalus, Sacch.pastorianus, Sacch. ellipsoideus and several unnamed bottom yeasts. He reported that only five out of thirty-seven cultures of wild yeast were unable to ferment trehalose. Unfortunately, it is not even possible to speculate as to the identity of these yeasts. Kluyver \& Roosmalen (1932) showed that Torula dattila Kluyver, and Saccharomyces cerevisiae Rasse van Delft fermented trehalose slowly, but Torula monosa Kluyver and Torula lactosa Kluyver were unable to do so. Myrbäck \& Örtenblad (1936) obtained variable results in tests on the fermentation of added trehalose by bakers' yeast; in some cases a slow fermentation occurred, whereas in others no gas was observed. On the other hand, dried yeast preparations made from samples of the same bakers' yeast fermented trehalose very well, indicating the presence of the enzyme system necessary for the fermentation of this sugar. Brewers' yeast (bottom yeast) was found to ferment added trehalose regularly but slowly.

We have checked the fermentation of yeast extract (autoclaved equal weights of water and bakers' yeast) by 133 cultures representing twenty genera and 
seventy-three species. The fermentation of added trehalose $(1 \%)$ in diluted yeast autolysate, prepared as previously described, was also examined. Most tests were repeated at least once. Of the large number of cultures used, only sixteen species, representing seven genera of yeasts, were positive for the fermentation of yeast extract and trehalose. In all cases, when the fermentation of plain yeast extract was positive, gas production in the autolysate plus added trehalose was also positive. There were some instances, however, when gas production occurred in the trehalose tubes but not in the yeast-extract tubes. When these tests were repeated with a more concentrated yeast extract and longer incubation period, gas production occurred in the yeast-extract tubes as well as the trehalose tubes. These fermentation tests are discussed in more detail in connexion with the various genera investigated.

Genus Candida. If trehalose fermentation has any value as a character in yeast classification it should be so with certain species in the genus Candida. This is particularly true of C. albicans and C.tropicalis, the definitions of which (Diddens \& Lodder, 1942) are quite broad and inclusive. Thirteen cultures of C. albicans, from various sources, were tested and seven produced gas in yeast extract as well as in the tubes with autolysate + trehalose. Six cultures were non-fermentative, two being cultures of Mackinnon's (1946) normal and variant strains. The variant, which was considered negative, showed a questionable fermentation in 13 days.

Two of three cultures of $C$. tropicalis gave positive fermentations. The identity of the culture which gave a negative fermentation is in our opinion questionable. The fermentation of trehalose by $C$. tropicalis is of particular interest, since strain N-18 and several other organisms isolated by us from cheese, olives, grapes, dates and soil, and capable of fermenting trehalose, are cultures of $C$. tropicalis, or are very similar to it. These are organisms that appeared to ferment both maltose and lactose in yeast-extract media, whereas actually they can ferment only maltose.

Two strains of $C$. stellatoidea and one stated to be $C$. deformans (probably C. lipolytica or $C$. zeylanoides), were positive for yeast extract, and trehalose. The following organisms grew, but failed to produce gas in either yeast extract, or autolysate plus trehalose: fourteen strains of $C$. krusei; five of $C$. chalmersi (C. parapsilosis); two of C.pseudotropicalis; and one strain each of C. suaveolens (C. humicola), C. guilliermondii, C. flareri (C. intermedia), C. intermedia, C. brumpti, C. zeylanoides var. macroglossia, C. parakrusei (C.parapsilosis) and C. pelliculosa.

Genus Saccharomyces. Sixty-three strains representing eleven species of this genus were tried. Only five strains were positive. In this genus the strongest fermenter of both yeast extract and trehalose was a hybrid, $S$. carlsbergensis $\times S$. cerevisiae obtained from $\mathrm{Dr} \mathrm{C}$. Lindegren. This is of interest since the four strains of $S$. carlsbergensis tested were negative and of the thirty-two strains of $S$. cerevisiae used, only one received from São Paulo, Brazil, was weakly positive. One strain each of $S$. italicus and $S$. cerevisiae Rasse fulliensis showed positive fermentation. In 1946 a culture of Saccharomyces, showing ability to ferment both maltose and lactose, was isolated from spoiled fruit. 
Unfortunately the culture was lost, but in all probability the unusual fermentation characteristic of this yeast was likewise an artefact.

The following were negative for the fermentation of yeast extract and trehalose: eight strains of $S$. cerevisiae var. ellipsoideus; four of $S$. tubiformis; three of $S$. oviformis; two of $S$. fragilis; two of $S$. chodati; and one strain each of $S$.exiguus, $S$. pastorianus, $S$. pyriformis and $S$. thermantitonum $(S$. cerevisiae var. ellipsoideus).

Subgenus Zygosaccharomyces. Seventeen strains representing twelve species were used. Only two strains, both $Z$. pini, were weakly positive. These strains were negative after $\mathbf{5}$ days in yeast extract prepared according to StellingDekker (1931); when a much more concentrated extract and twelve days' incubation were used they were positive. Organisms not showing fermentation were: two strains of $\boldsymbol{Z}$. barkeri; two of Z.japonicus var. Soya; two of Z. bisporus; and one strain each of $Z$. marxianus, $Z$. nadsonii, Z. pastori, Z. acidifaciens, Z. globiformis, $Z$. richteri and $Z$. lactis.

Genus Hansenula. Of thirty-seven strains representing seven species only five strains were fermenters of trehalose. After 10 days of incubation only one of six strains of $\boldsymbol{H}$. anomala and three out of nine strains of $\boldsymbol{H}$. anomala var. long $a$ were positive. The results obtained with the variety 'longa' were quite variable, depending on the concentration of yeast extract and period of incubation. Similar results were obtained with a single strain of $\boldsymbol{H}$. ciferri. 'Those showing no fermentation were seven strains of $H$. anomala var. spherica; six of $\boldsymbol{H}$. saturnus; three of $\boldsymbol{H}$. subpelliculosa; and one strain each of $\boldsymbol{H}$. schnegii, H. lambica and H. anomala var. heteromorpha.

Genus Debaryomyces. Only three strains were tried but two (D. globosus and $D$. matruchoti) were quite strongly positive, whereas $D$. tyrocola was negative.

Torulaspora. A single strain, $T$. fermentati was positive, and one of $T$. delbrücki negative.

Nematospora. $N$. coryli (one strain) was one of the strongest gas-producers in yeast extract as well as in trehalose.

Torulopsis. Eight strains representing eight species were tried. Only $T$. alactosa ( $T$. holmii Rasse Delft) was unquestionably positive. $T$. dattila produced a few small bubbles. Kluyver \& van Roosmalen (1932) also reported this to be a slow fermenter of trehalose. Those showing no fermentation were: two strains of $T$. pulcherrima (C.pulcherrima); T.fermentans; T. kefir; T. monosa; T. californicus.

Genus Brettanomyces. A single strain identified as B. bruxellensis failed to show any fermentation. However, two strains isolated from Irish beer and tentatively identified as Brettanomyces species fermented both yeast extract and trehalose rapidly.

Other genera. All other strains tried were negative. These were: Schizosaccharomyces octosporus and Sch. pombe; Saccharomycoides ludwigii; Endomycopsis selenospora, E. albicans, E. capsularis and E. lindneri; Endomyces magnusii; Schwanniomyces occidentalis; Pichia chodati, P fermentans, $\boldsymbol{P}$. kluyveri and P. membranaefaciens; Zygopichia chevalieri; Zygohansenula californica; 
Nadsonia fulvescens; Eremothecium ashbyi; Schizoblastosporion. starkeyi-henricii and Kloeckera africana and $K$. lindneri.

The authors are indebted to Dr Carl Lindegren for the culture of Saccharomyces cerevisiae $\times$ carlsbergensis, to Dr Juan Mackinnon for the normal and variant strains of Candida albicans, and to Dr L. Wickerham for several of the other strains used.

\section{REFERENCES}

BAU (1899). Über Gärversuch mit Trehalose. Wochenschrift für Brauerei. (Cf. Lafar (1910), Tech. Mycology, II. London: Griffin and Co.)

BEDFORD, C. L. (1942). A taxonomic study of the genus Hansenula. Mycologia, 34, 628-49.

Brandt, K. M. (1941). Biochem. Z. 309, 190-201. (Cited through Chem. Abstr.)

Custers, M. TH. J. (1940). Onderzoekingen over Het gistgeslacht Brettanomyces, pp. 1-178. Thesis, Delft, Holland.

Diddens, H. A. \& Lodder J. (1942). Die Hefesammlung des 'Centraalbureau voor Schimmelcultures'. II. Teil. Die Anaskosporogenen Hefen, Zweite Hälfte, pp. 1-511. Amsterdam. N.V. Noord Hollandsche Uitgevers Maatschappij.

Henrici, A. T. (1941). The yeasts. Bact. Rev. 5, 97-179.

KuUyver, A. J. (1931). Quelques remarques sur la classification des levures. Ann. Zymologie, Sér. II, 1, 48-61.

Kluyver, A. J. \& van Roosmalen, F. L. W. (1932). Fermentation of trehalose. Biochem. Z. 245, 13-24.

Koch, E. \& Koch, F. C. (1925). Presence of trehalose in yeast. Science, 61, 570-2.

Lindner (1887). Wochenschrift für Brauerei, 4, 853. (Cf. Lafar (1910). Tech. Mycology, II. London: Griffin and Co.)

Lodder, J. (1934). Die Hefesammlung des 'Centraalbureau voor Schimmelcultures'. II. Teil. Die Anaskosporogenen Hefen, Erste Hälfte. Verh. Akad. Wet. Amst. Afdeel. Natuurkunde, 2nd sect. 32, 1-256.

Mackinnon, J. E. (1946). Zimologia Medica, pp. 1-160. Montevideo: El Siglo Illustrado.

Morrow, C. A. \& Sandstrom, W. M. (1935). Biochemical Laboratory Methods for Students of the Biological Sciences, 2nd ed., 319 pp. New York: J. Wiley and Sons.

Müntz, A. (1876). Recherches sur les fonctions des champignons. Ann. Chim. Phys. $5^{\mathrm{e}}$ Sér. 8, 56-92.

Myrbäck, K. (1937). Yeast and trehalose. Svensk. Kem. Tidskr. 49, 124-7.

Myrbäck, K. \& Örtenblad, B. (1936). Trehalose und Hefe. I. Biochem. Z. 288, $329-37$.

Myrbäck, K. \& Örtenblad, B. (1937). Trehalose und Hefe II. Trehalasewirkung von Hefepräparaten. Biochem. Z. 291, 61-9.

Rhoades, H. E. (1941). The adaptive enzymes of certain strains of yeast. J. Bact. 42, 99-115.

SACChETt, M. (1933). Contributo alla conoscenza della flora microbica di alcuni formaggi italiani. Arch. Mikrobiol. 4, 427-45.

Steiner, A. \& Cori, C. F. (1935). The preparation and determination of trehalose in yeast. Science, 82, 422-3.

Stelling-Dekker (1931). Die Hefesammlung des 'Centraalbureau voor Schimmelcultures'. I. Teil. Die sporogenen Hefen. Verh. Akad. Wet. Amst. Afdeel. Natuurkunde, 2nd sect. Deel 28, no. 1.

Tanket, G. (1931). The trehalose of yeasts. C.R. Acad. Sci., Paris, 192, 1056-8. 